\title{
Estimate of the rate of unreported COVID-19 cases during the first outbreak in Rio de Janeiro
}

\author{
M.S. Aronna ${ }^{1}$, R. Guglielmi ${ }^{2, *}$ L.M. Moschen ${ }^{1}$
}

\section{Abstract}

In this work we fit an epidemiological model SEIAQR (Susceptible - Exposed - Infectious - Asymptomatic - Quarantined - Removed) to the data of the first COVID-19 outbreak in Rio de Janeiro, Brazil. Particular emphasis is given to the unreported rate, that is, the proportion of infected individuals that is not detected by the health system. The evaluation of the parameters of the model is based on a combination of error-weighted least squares method and appropriate B-splines. The structural and practical identifiability is analyzed to support the feasibility and robustness of the parameters' estimation. We use the bootstrap method to quantify the uncertainty of the estimates. For the outbreak of March-July 2020 in Rio de Janeiro, we estimate about $90 \%$ of unreported cases, with a $95 \%$ confidence interval $(85 \%, 93 \%)$.

Keywords: COVID-19 model, parameter estimation, identifiability, least squares, B-splines, bootstrap method

\footnotetext{
${ }^{*}$ Corresponding author

Email addresses: soledad.aronna@fgv.br (M.S. Aronna), roberto.guglielmi@uwaterloo.ca (R. Guglielmi), lucas.moschen@fgv.edu.br (L.M. Moschen)

${ }^{1}$ Escola de Matemática Aplicada - EMAp, FGV, Rio de Janeiro, RJ, Brazil

${ }^{2}$ Department of Applied Mathematics, University of Waterloo, Waterloo, ON, Canada
} 


\section{Introduction}

2

In late December 2019, health professionals in the city of Wuhan (Hubei, China) identified several cases of pneumonia (The 2019 nCoV Outbreak Joint Field Epidemiology Investigation Team and Q. Li, 2020) caused by a new coronavirus, which was named SARS-CoV-2. The disease induced by SARSCoV-2, called COVID-19, rapidly spread around the world. Most COVID19 cases are asymptomatic patients or with mild symptoms, but in more severe cases the disease may progress to viral pneumonia and multi-organ failure (World Health Organization, 2020) and can lead to hospitalization and death.

Brazil declared the disease as a public health emergency in February 2020, before the first COVID-19 cases were reported in Brazil. With the aim of protecting the population, several measures were implemented, including the adjustment of a legal framework to carry out isolation and quarantine Croda et al., 2020). In March 2020, the governor of the state of Rio de Janeiro declared a public health emergency and ordered to avoid gatherings, closing down schools, restaurants, and theaters, and restricting access to beaches, shopping centers, and non-essential commerce (Dantas et al., 2020). Nevertheless, these measures could not prevent the outbreak of the virus in the region, fueled by the large portion of asymptomatic individuals and by the long incubation period of the virus (Li et al., 2020). The objective of this work is to provide a quantitative estimate of crucial parameters related to the COVID-19 outbreak in the city of Rio de Janeiro during the period March-July 2020. The analysis is based on the fitting of the SEIAQR compartmental model introduced in (Aronna et al., 2021) to real data retrieved 
from the public health agency (Municipal Health Department, City Hall of Rio de Janeiro, 2021). This epidemiological model takes into account isolation, quarantine of confirmed cases, and testing of asymptomatic individuals as non-pharmaceutical strategies to contain the spread of the virus among the population (more details in Section 2.1). The main purpose of the study is to estimate the rate of unreported cases in the city of Rio de Janeiro. This is a crucial step to gauge the real extent and impact of the disease in the population, since most of the infections do not result in severe symptoms and are therefore likely to remain undetected.

The text is organized as follows: Section 2 collects different information regarding the mathematical model; the structure of the data as retrieved for the city of Rio de Janeiro; and the representation chosen for the parameters' estimation. Section 3 describes the notions of identifiability analyzed in the paper. Section 4 presents the results of the fitting of the data to the model and the resulting estimates of the parameters. Finally, the last two sections conclude the paper with some discussions and final comments.

\subsection{Data Availability}

No new data are released as part of this research. This paper relies on publicly available datasets, with references provided in the text. The code required to reproduce our analysis is available in the GitHub repository (Moschen, 2021). 


\section{Material and methods}

\subsection{The epidemiological model}

q We recall the key features of the compartmental model from Aronna et al., 2021). The population is split into the compartments $S, E, I, A, Q$, $R$, and $D$, corresponding to the compartments of susceptible, exposed, infectious, infectious and asymptomatic, quarantined or hospitalized, recovered, and dead individuals, respectively. These compartments are linked in the following way (see Figure 1): the individuals in $S$ move to compartment $E$ when exposed to the virus. After a given latent period, they become infectious and thus pass to compartment $I$. At this stage, an individual in $I$ may either report symptoms and thus move (after testing) to the compartment $Q$, or result in an asymptomatic/paucisymptomatic infection and move to the compartment $A$. Individuals from $E$ and $A$ may move to $Q$ as a result of the testing strategy among the asymptomatic population, as described in more detail in the following. Finally, the compartment $R$ collects the recovered individuals from either $A$ or $Q$, whereas the compartment $D$ counts the COVID-19-related deaths. By normalizing the total population to 1, the value of each variable $S, E, I, A, Q, R$, and $D$ represents the proportion of that given compartment in the total population. Moreover, we neglect the birth and natural death rates, given the limited time horizon chosen for the data fitting. The dynamics is described by the following set of differential 


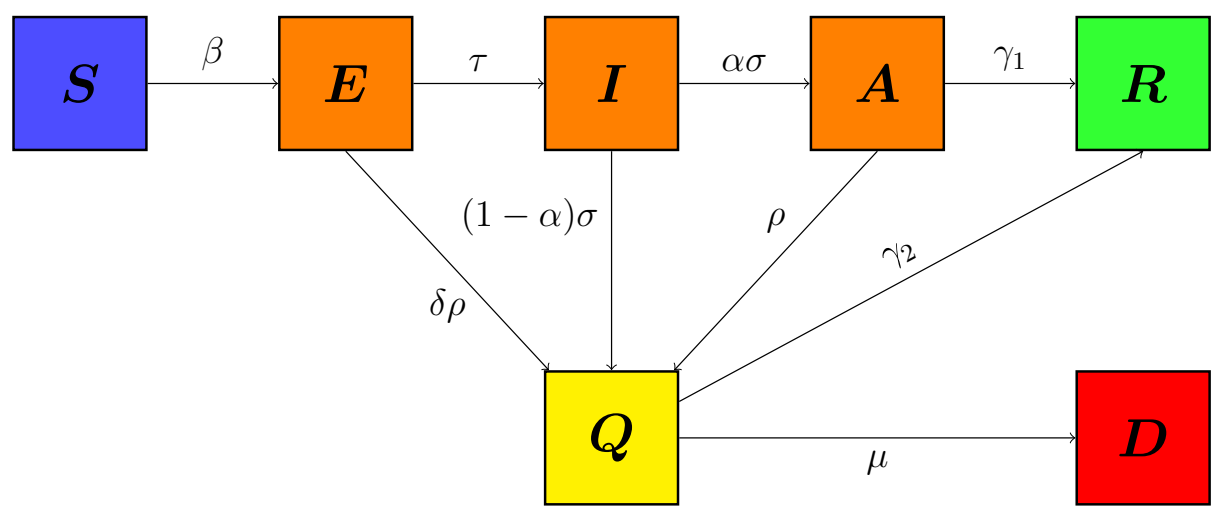

Figure 1: Diagram of the compartmental model

68

equations:

$$
\begin{aligned}
& \dot{E}=\beta(t) S(I+A)-\rho \delta E-\tau E \\
& \dot{I}=\tau E-\sigma I-\rho I \\
& \dot{A}=\sigma \alpha I-\rho A-\gamma_{1} A \\
& \dot{Q}=\sigma(1-\alpha) I+\rho(\delta E+I+A)-\gamma_{2} Q-\mu(t) Q \\
& \dot{S}=-\beta(t) S(I+A) \\
& \dot{R}=\gamma_{1} A+\gamma_{2} Q \\
& \dot{D}=\mu(t) Q,
\end{aligned}
$$

which is a simplified version of the model studied in (Aronna et al., 2021). More precisely, the simplification is done by considering the population as a sole homogeneous group that is subject, in average, to the same mobility restrictions, while the model from (Aronna et al. 2021) allowed for a distinction between two groups - one with high mobility consisting of essential workers and the other with restricted mobility composed by the remainder of the population. The parameters $\tau, \sigma, \omega, \gamma_{1}, \gamma_{2}$, and $\mu$ related to the pathogen 
and the induced disease are described in Table 1. The function $\beta(t)$ is the effective contact rate at time $t$, which takes into account the average contact rate - directly affected by social distancing, public policies, use of Personal Protective Equipment (PPE), etc. - and the transmissibility of the virus probability of infection given a contact between an infected and a susceptible individual. A constant of particular interest in our analysis is the parameter $\alpha \in(0,1)$, which represents the proportion of asymptomatic infectious cases. Among these cases, only those found positive through either testing induced by contact tracing or simply random testing are reported to the health system. All the others will remain unreported, being paucisymptomatic (but infectious) cases. In view of the scarcity of testing kits during the first outbreak in Rio de Janeiro, we consider the parameter $\alpha$ as a proxy for the unreported rate of infections. It is clear that estimating such parameter $\alpha$ is crucial to assess the effective size of the epidemic since such unreported cases may fuel the ongoing outbreak. Moreover, having an approximation of the size of the recovered population is also an essential information for epidemiological management and the comprehension of the virus behaviour. At the same time, estimating $\alpha$ may be very difficult, since it accounts for those cases not detected by the radar of the health system (Nogrady, 2020).

As mentioned above, the unreported rate is strictly related to the availability of testing kits and to the effectiveness of tracing, tracking, and testing in place during the outbreak. Such measures are described in model (1) by both the detection rate $1-\alpha$ and the parameter $\rho$, the latter representing the rate of testing among asymptomatic or paucisymptomatic individuals. We point out that the value of the unreported rate $\alpha$ is not sensitive to small 
variations of values of $\rho$ (see Section 2.3.1).

For clarity of exposition, in model (1) we do not describe important features such as the sensitivity and the specificity of the testing kits, although these attributes may play a crucial role in consideration of new variants of the virus (see (Aronna et al., 2021, Remark 2.3) for more details). With this premise, in our model we assume that a person in the compartments $I$ or $A$ will always test positive, in $S$ always negative, and in $E$ positive with a probability $\delta \in(0,1)$. We also introduce the counter $T(t)$ of total positive tests, which follows the dynamics

$$
\dot{T}=\sigma(1-\alpha) I+\rho(\delta E+I+A) .
$$

\begin{tabular}{|c|c|}
\hline Par. & Description \\
\hline$\tau^{-1}$ & latent period, from exposure to infectiousness \\
\hline$\sigma^{-1}$ & time from infectiousness to possible symptoms onset \\
\hline$\omega^{-1}$ & incubation period (i.e. $\left.\omega^{-1}=\tau^{-1}+\sigma^{-1}\right)$ \\
\hline$\gamma_{1}$ & recovery rate for paucisymptomatic individuals \\
\hline$\gamma_{2}$ & recovery rate for detected positive cases \\
\hline$\mu$ & mortality rate among detected positive cases \\
\hline
\end{tabular}

Table 1: Parameters of COVID-19

\subsubsection{The basic reproduction number}

Assuming a constant contact rate $\beta$, the basic reproduction number $\mathcal{R}_{0}$ associated with the model (11) is given by the relation 


$$
\mathcal{R}_{0}=\frac{1}{2}\left(\varphi+\sqrt{\varphi^{2}+\frac{4 \sigma \alpha}{\rho+\gamma_{1}} \varphi}\right)
$$

where

$$
\varphi=\frac{\beta \tau}{(\rho \delta+\tau)(\sigma+\rho)}
$$

\section{1, 2020 to July 31, 2020. The format of the data is displayed in Table 2. The}

\begin{tabular}{llllllll}
\hline Notification date & Symptom onset date & Neighborhood & Sex & Age group & Outcome & Outcome date & Ethnicity \\
\hline $05 / 18 / 20$ & $05 / 03 / 20$ & PACIENCIA & M & $50-59$ & death & $05 / 17 / 20$ & Black \\
$04 / 25 / 20$ & $04 / 02 / 20$ & BARRA DA TIJUCA & M & $80-89$ & death & $05 / 01 / 20$ & White \\
$05 / 06 / 20$ & $05 / 06 / 20$ & CACHAMBI & M & $70-79$ & death & $05 / 07 / 20$ & Ignored \\
$06 / 12 / 20$ & $06 / 02 / 20$ & BARRA DA TIJUCA & M & $70-79$ & death & $06 / 12 / 20$ & White \\
$06 / 13 / 20$ & $04 / 26 / 20$ & MARECHAL HERMES & M & $60-69$ & death & $06 / 16 / 20$ & Ignored \\
\hline
\end{tabular}

Table 2: Extract of the data: notification date, symptom onset date, neighborhood of residence, sex, age group, outcome, outcome date, ethnicity. 
column Symptom onset date denotes the symptom onset date reported by the patient, and Outcome date marks the date on which the person recovers or dies (respectively recovered or death in the column Outcome), thus ceasing to be an active case. The column Outcome date was incomplete having about $3.7 \%$ of empty fields, so we were not able to use the recovery date as an input in our data fitting and missing death dates were imputed randomly according to the empirical distribution of the time difference between notification and evolution dates. Finally, we normalize the data by the size of the population in Rio de Janeiro, which we assume to be 6.7 millions (IBGE, 2021).

\subsubsection{Smoothed and cumulative curves}

There is a weekly seasonality in confirmed cases and deaths in the outbreak with a negative deviation on weekends. This variation affects the model since it has no seasonal adjustment. For this reason, the data is smoothed using a centered moving average with 7 days. Thus, given a set of initial data $\left\{x_{s}\right\}_{1 \leq s \leq n}$ of confirmed positive cases or deaths, we replace it with

$$
\hat{x}_{t}=\frac{1}{2 k+1} \sum_{s=-k}^{k} x_{t+s},
$$

where $2 k+1=7$. Although this choice may seem arbitrary, it is directly related to the weekly periodicity of the data. Figures 2 and 3 display daily new cases and deaths, respectively. In particular, in Figure 2 we can observe that the first wave ends at the end of July, which justifies our choice to set the horizon of the data fitting on July 31st. Moreover, we calculate the curves of cumulative positive cases and deaths by evaluating 


$$
y_{t}=\sum_{i=-14}^{t} \hat{x}_{i}, \quad(t=0, \ldots, n) .
$$

The curves of cumulative cases, considering the self-reported symptoms onset, and cumulative deaths will be compared to the compartments $T$ and $D$ from model (1).

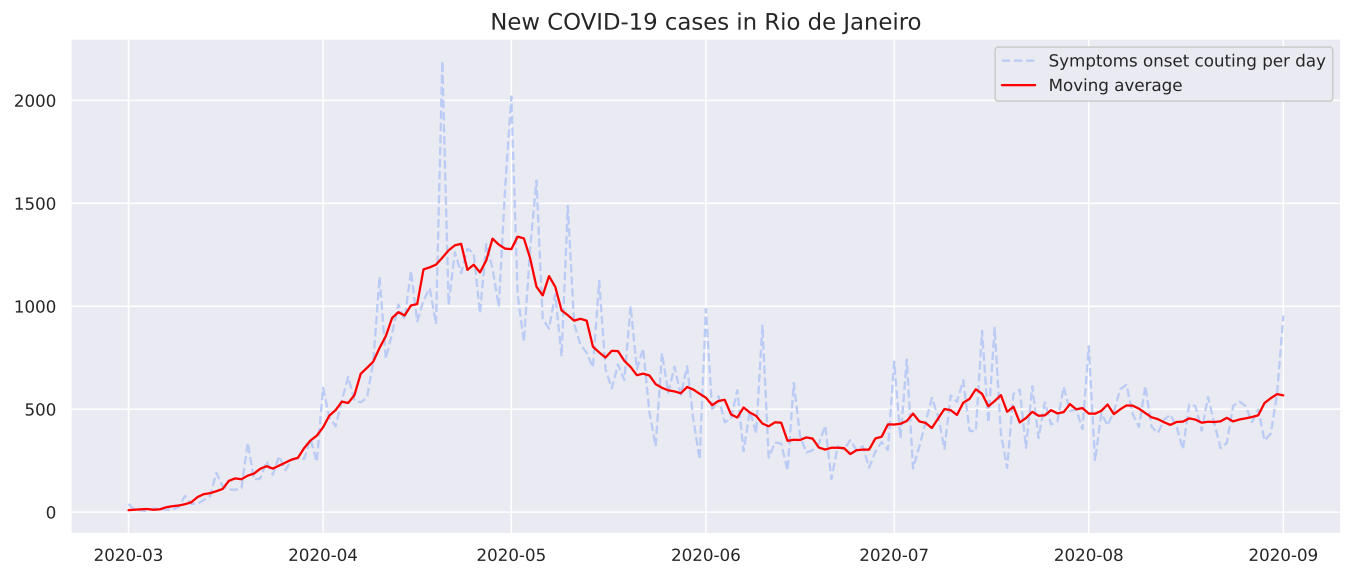

Figure 2: Daily new cases considering the self-reported symptoms onset in Rio de Janeiro between March and September of 2020. The 7-days moving average is displayed in red.

\subsection{Choice and modeling of parameters}

We split the set of parameters between a first group, consisting of epidemiological constants $\tau, \sigma, \gamma_{1}, \gamma_{2}$, and $\delta$, whose values we retrieve from the literature (see Table 3), and a second group of parameters $\beta(t), \alpha, \rho$, and $\mu(t)$ estimated from the data. The model used to represent the effective contact rate $\beta(t)$ and the mortality $\mu(t)$ is described in Section 2.3.2. We start by estimating the testing rate $\rho$. 


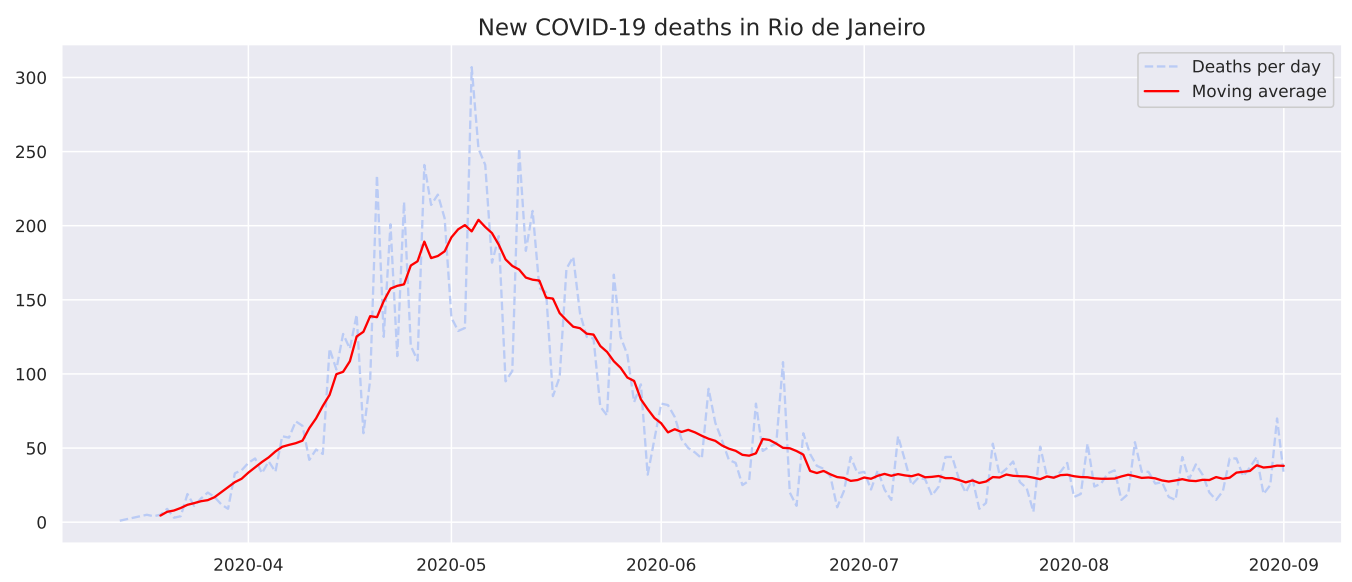

Figure 3: Daily new COVID-19 related deaths in Rio de Janeiro between March and September of 2020. The 7-days moving average is displayed in red.

\subsubsection{Estimation of $\rho$}

We consider the testing data from the state of Rio de Janeiro (IBGE, 2020), summarized in Table 4: about $2 \%$ of the population is tested each month, with a positive rate of about $20 \%$ among the tests done. We thus evaluate a daily testing of about $0.013 \%$ of the population, that is, we shall choose $\rho \leq 1.3 \cdot 10^{-4}$. In this range of values, the impact of the choice of $\rho$ on $\alpha$ is negligible, as we verify in Table 6. For this reason, we assign to $\rho$ the value of $\rho=10^{-5}$. Unfortunately, the data do not allow to discern the tests due to symptom onset from the testing of asymptomatic cases, due to the effectiveness of the trace, track, and test strategy.

\subsubsection{Representation of $\beta$ and $\mu$}

The parameter $\beta$ in model (1) varies in time according to the different public policies in place in Rio de Janeiro in different periods (Official Journal of the State of Rio de Janeiro, 2020b; Estadão, 2020), and according to 


\begin{tabular}{|c|c|c|c|}
\hline Par. & Value & \multicolumn{2}{c|}{ Reference } \\
\hline$\omega^{-1}$ & 5.74 days & Rai et al. & $(2021)$ \\
\hline$\tau^{-1}$ & 3.69 days & Li et al. & $(2020)$ \\
\hline$\sigma^{-1}$ & $\omega^{-1}-\tau^{-1}$ & Li et al. & $(2020)$ \\
\hline$\gamma_{1}^{-1}$ & 7.5 days & Byrne et al. & $(2020)$ \\
\hline$\gamma_{2}^{-1}$ & 13.4 days & Byrne et al. & (2020) \\
\hline$\delta$ & 0.01 & Kucirka et al. & 2020) \\
\hline
\end{tabular}

Table 3: Value of parameters retrieved from the literature.

\begin{tabular}{|c|c|c|c|c|}
\hline & July & August & September & October \\
\hline Percentage (\%) & 6.8 & 8.6 & 10.2 & 11.9 \\
\hline Positive rate (\%) & 1.2 & 1.5 & 1.9 & 2.4 \\
\hline
\end{tabular}

Table 4: Percentage of cumulative total and positive tests among the population.

the compliance of the population with these measures. For this reason, we approximate $\beta$ by $B$-splines in the form

$$
\beta(t) \approx \sum_{j=1}^{s} \beta_{j} B_{j, k}(t),
$$

where $\beta_{j}$ are the coefficients to be estimated and $\left\{B_{j, k}(t)\right\}_{j=1}^{s}$ is the basis of functions of order $k$ (De Boor, 1978). A similar representation is used for the mortality rate $\mu(t)$

$$
\mu(t) \approx \sum_{j=1}^{r} \mu_{j} B_{j, k}(t),
$$

which has to be understood as relative to the number of confirmed deaths. This reflects the fact that the relative mortality may vary in periods of dis- 
tress for the health system and lack of testing kits. We therefore define the vector of $s+r+1$ parameters to be estimated

$$
\theta=\left(\alpha, \beta_{1}, \ldots, \beta_{s}, \mu_{1}, \ldots, \mu_{r}\right)
$$

We assume that the knots, i.e. the time points where the polynomials connect, are equally spaced. The number of knots is equal to the number of parameters ( $s$ for $\beta$ and $r$ for $\mu$ ) plus the order of the B-spline $(k)$ plus 1.

To determine the order $k$ and the number of coefficients $s, r$, for the Bspline approximation, we use the Akaike Information Criterion (AIC) (Liang et al. 2010), which under the normality hypothesis of the errors is given by the formula

$$
A I C=n \ln \left(\frac{R S S}{n}\right)+2(s+r+1),
$$

such that $R S S$ is the sum of squares of the model's residuals. For the numerical experiments in Section 4, we compare models with 3 and 4 coefficients, as models with less than 3 coefficients are unable to capture temporal variations over the timeframe of the outbreak, while models with $s$ and $r$ greater than 4 are computationally difficult to handle.

\subsection{Parameter estimation}

ـ For the parameter estimation, we follow the methods applied in Cao et al., 2012; Liang et al., 2010, Ramsay et al., 2007). The first 15 days of March were used to estimate the initial conditions (see Section 2.4.1) and thus set up the model starting from March 16th, when the first containment measures were imposed Official Journal of the State of Rio de Janeiro, 
2020a). Denoting by $\hat{x}_{i}^{(1)}$ and $\hat{x}_{i}^{(2)}$ the daily new cases and deaths, respectively, on the $i$-th day, where $i=0$ and $i=n$ indicate March 16th and July 31 th, respectively, we assume that

$$
\begin{aligned}
\hat{x}_{i}^{(1)} & =(T(i)-T(i-1))+\varepsilon_{i}^{(1)}, \quad i=-14, \ldots, n, \\
\hat{x}_{i}^{(2)} & =(D(i)-D(i-1))+\varepsilon_{i}^{(2)}, \quad i=-14, \ldots, n,
\end{aligned}
$$

where the sequences $\left\{\varepsilon_{i}^{(1)}\right\}_{-14 \leq i \leq n}$ and $\left\{\varepsilon_{i}^{(2)}\right\}_{-14 \leq i \leq n}$ are independent and normally distributed random variables with unknown variances $\sigma_{k}^{2}, k=1,2$. Summing up we get

$$
\begin{array}{ll}
y_{i}^{(1)}=T(i)+\xi_{i}^{(1)}, & i=0, \ldots, n, \\
y_{i}^{(2)}=D(i)+\xi_{i}^{(2)}, & i=0, \ldots, n,
\end{array}
$$

for the cumulative quantities, where $\xi_{i}^{(k)}=\sum_{j=-14}^{i} \varepsilon_{j}^{(k)}$ for $k=1,2$. Therefore, for any $i \leq j \leq n$ and $k=1,2$, the covariance matrix is defined by

$$
\operatorname{Cov}\left(\xi_{i}^{(k)}, \xi_{j}^{(k)}\right)=(i+15) \sigma_{k}^{2}
$$

At this point, we introduce the notations $\hat{T}(\theta)$ and $\hat{D}(\theta)$ for the numerical approximations of the functions $T$ and $D$, obtained by integrating equations (2) and (1), respectively, through the Runge-Kutta method and corresponding to the parameter vector $\theta$ introduced in (4).

\subsubsection{Initial values estimation}

Considering $S \approx 1$ in the first 15 days of the epidemic, and focusing on the compartments $E, I, A$, and $T$, the system (1) reduces to 


$$
\left[\begin{array}{c}
\dot{E} \\
\dot{I} \\
\dot{A} \\
\dot{T}
\end{array}\right]=\left[\begin{array}{cccc}
-\tau & \beta & \beta & 0 \\
\tau & -\sigma & 0 & 0 \\
0 & \sigma \alpha & -\gamma_{1} & 0 \\
0 & \sigma(1-\alpha) & 0 & 0
\end{array}\right] \cdot\left[\begin{array}{c}
E \\
I \\
A \\
T
\end{array}\right],
$$

whose solution is a linear combination of exponential functions. The daily testing of asymptomatic individuals at the beginning of the pandemic is assumed to be negligible, thus $\rho \approx 0$, while the parameters $\gamma_{1}, \tau$, and $\sigma$ are taken from the existing literature (see Table 3). The parameters $\alpha$ and $\beta$ are fixed in this period. The value $T(-14)$ corresponds to the confirmed cases on March 2 and $T(-15)$ is set to zero. Therefore, the parameters to be estimated in this period are reduced to $\theta_{0}=(\alpha, \beta, E(-14), I(-14), A(-14))$. Considering $\hat{T}\left(\theta_{0}\right)_{i}$ the approximation for $T$ as function of $\theta_{0}$ at day $i$, we aim to minimize the expression

$$
\sum_{i=-14}^{0} w_{i}\left(y_{i}^{(1)}-\hat{T}\left(\theta_{0}\right)_{i}\right)^{2},
$$

where the weights $w_{i}=\frac{i+14}{14}$ are chosen to give less importance to the initial days. With these estimates, we obtain the values $(E(0), I(0), A(0))$. Since $R(-14)=0$, we get that $Q(-14)=T(-14)$, and thus we can integrate the curves $Q$ and $R$ to obtain the values $Q(0)$ and $R(0)$. Finally, we deduce $S(0)=1-E(0)-I(0)-A(0)-Q(0)-R(0)$.

Remark 2.1. In order to analyze the sensitivity of the curves $\hat{T}$ and $\hat{D}$ with respect to the initial guess of the parameter $\theta_{0}$, we perform a series of random realizations and compare the final values $\hat{T}\left(\theta_{0}\right)_{n}$ and $\hat{D}\left(\theta_{0}\right)_{n}$ for all guesses. 


\subsubsection{Curve fitting}

We use the weighted least squares method to estimate the unknown parameters by the data of daily confirmed cases and deaths given by equations (7)-(8). This approach is based on the solution of a constrained nonlinear minimization problem. We consider the following objective functional

$$
F(\theta)=\left(y^{(1)}-\hat{T}(\theta)\right)^{T} \Sigma^{-1}\left(y^{(1)}-\hat{T}(\theta)\right)+\psi\left(y^{(2)}-\hat{D}(\theta)\right)^{T} \Sigma^{-1}\left(y^{(2)}-\hat{D}(\theta)\right)
$$

where $M^{T}$ denotes the transpose matrix of $M, \sigma_{1}^{2} \Sigma$ and $\sigma_{2}^{2} \Sigma$ are the covariance matrices given by equations (9), and $\psi$ is a weight proportional to the ratio of the variances $\sigma_{1}^{2} / \sigma_{2}^{2}$. We solve the minimization problem by means of the L-BFGS-B algorithm (Byrd et al., 1995), which combines the gradient projection method and the BFGS algorithm with optimized use of computational memory, implemented in the SciPy library in Python (Virtanen et al. $2020)$.

\subsection{Uncertainty quantification over the parameters}

Uncertainty is intrinsic in the parameters' estimation, owing to a combination of different factors, such as the natural variability of the data, the measurement errors of the data collection, and the biases of the estimation method. In order to tackle this problem, we construct confidence intervals for each unknown parameter. We rely on the Bootstrap method (Efron and Tibshirani, 1986), which involves a constructive approach based on the data. Starting from a series $Y$, it generates replicated data $Y_{1}^{*}, \ldots, Y_{N}^{*}$ and performs the estimations for each one. The confidence interval for the value 
of interest is then given by the corresponding percentile of the $N$ replicated samples (Joshi et al., 2006).

As a consequence of the error structure presented in equation (5), we generate each replicated curve $T^{(B)}$ with the initial value $T^{(B)}(0)=y_{0}^{(1)}$ and satisfying $T^{(B)}(i+1)=T^{(B)}(i)+\varepsilon_{i+1}$ for any $i \geq 1$, where $\varepsilon_{i+1} \sim \mathcal{N}\left(\hat{x}_{i+1}^{1}, \hat{\sigma}_{1}^{2}\right)$ and $\hat{\sigma}_{1}^{2}$ (see Appendix A is an estimate for $\sigma_{1}^{2}$. An analogous construction is carried out for equation (6). In the estimation process, in order to avoid local minima, we randomize the initial guess of the optimization algorithm: for every $j=1, \ldots, r+s+1$, we select initial guesses $\theta_{j}^{\text {initial }}$ randomly chosen with uniform distribution in a prescribed range $\left(l_{j}, u_{j}\right)$, that represents the interval of admissible values for that parameter. After $m$ iterations of this process, we pick the best solution minimizing the objective functional (10).

\section{Theory}

\subsection{Identificability}

From a structural-theoretical point of view, we are interested in analyzing the identifiability of model (1). In general, this analysis identifies whether unknown parameters can be estimated in a unique way from the available measurements on the system Audoly et al., 2001; Saccomani and Thomaseth, 2019). There are two conceptually different ways to develop this analysis: the structural approach (a priori) and the practical approach (a posteriori). The former is a theoretical property that resides in the structure of the model itself: the parameters are structurally identifiable if they can be (globally) uniquely identified from the available measurements; they are called locally structurally identifiable if they are structurally identifiable 
within a neighbourhood of the solution. On the other hand, the practical approach is based on the outcome of the data fitting, and it is evaluated by means of the correlation matrix of the parameters.

\subsection{Structural identifiability}

Consider a dynamical system

$$
\begin{aligned}
\dot{x} & =f(x(t), \theta), \quad x(0)=x_{0} \\
y(t) & =h(x(t), \theta)
\end{aligned}
$$

where $x(t) \in \mathbb{R}^{n}, y(t) \in \mathbb{R}^{m}$ and $f$ and $h$ are rational functions of the variable $x$ and $\theta \in \Theta \subset \mathbb{R}^{p}, \Theta$ being the set of admissible values of the parameter $\theta$. The variable $y$ is the output of the system, that is the observable component. In the model, $y$ is the number of confirmed cases and deaths. When $x(0)=$ $x_{0}$, the measurement with initial value $x_{0}$ is $y=\psi_{x_{0}}(\theta)$. The structural identifiability (Ljung and Glad, 1994) of system (11) at a fixed $\theta^{*} \in \Theta$ is related to the number of solutions of the equation

$$
\psi_{x_{0}}(\theta)=\psi_{x_{0}}\left(\theta^{*}\right)
$$

The system (11) is globally identifiable at $\theta^{*}$ if equation 12 has a unique solution $\theta=\theta^{*}$, or, equivalently if the mapping $\psi_{x_{0}}$ is invertible. System (11) is locally identifiable if $\psi_{x_{0}}$ is invertible in a neighborhood of $\theta^{*}$. In Appendix B we describe a computational method to verify structural identifiability using the DAISY software (Bellu et al., 2007).

\subsection{Practical identifiability}

The structural identifiability process developed in Section 3.2 is based on two hypotheses: the model structure is accurate and measurement errors 
are absent. These hypotheses are not valid in practice and, therefore, it is necessary to assess whether the parameters can be reliably and accurately estimated from noisy data (Miao et al. 2011). Let $\hat{\theta}=\left(\hat{\alpha}, \hat{\beta}_{1}, \ldots, \hat{\mu}_{r}\right)$ be the vector of parameters estimated from the data fitting, and $\hat{T}, \hat{D}, \hat{\sigma}_{1}$ and $\hat{\sigma}_{2}$ the model approximations for the confirmed cases, deaths, and their respective estimated variances, as introduced in Section 2.4. The correlation matrix quantifies the interdependence between model parameters, and can be computed as follows: starting from the Fisher Information matrix (FIM)

$$
F I M=\left.\left.\frac{1}{\hat{\sigma}_{1}^{2}}\left(\frac{\partial \hat{T}}{\partial \theta}\right)\right|_{\theta=\hat{\theta}} ^{T} \Sigma^{-1}\left(\frac{\partial \hat{T}}{\partial \theta}\right)\right|_{\theta=\hat{\theta}}+\left.\left.\frac{1}{\hat{\sigma}_{2}^{2}}\left(\frac{\partial \hat{D}}{\partial \theta}\right)\right|_{\theta=\hat{\theta}} ^{T} \Sigma^{-1}\left(\frac{\partial \hat{D}}{\partial \theta}\right)\right|_{\theta=\hat{\theta}},
$$

we compute the covariance matrix $C$ as the inverse of FIM. Then, the element $r_{i j}, 1 \leq i, j \leq r+s+1$ of the correlation matrix $R$ is defined as

$$
r_{i j}=\frac{C_{i j}}{\sqrt{C_{i i} C_{j j}}}
$$

which measures the correlation between $\hat{\theta}_{i}$ and $\hat{\theta}_{j}$. A value close to 1 indicates that the parameters are strongly interconnected, and that each of them varies according to the other.

\section{Results}

We first set the initial conditions according to Section 2.4.1 and we approximate the curve of the first two weeks of the outbreak, as represented in Figure 4. Following Remark 2.1, the fitting with respect to the initial guess for $\theta_{0}$ is robust: the final values on July 31st range from 0.0127 to 0.0131 . As summarized in Table 5, the best fitting over the outbreak period March-July 

parameters $\beta$ and $\mu$, and B-spline of order 2 for $\beta$ and order 1 for $\mu$.

\begin{tabular}{|c|c|c|c|c|c|c|c|c|}
\hline \multirow{2}{*}{\multicolumn{2}{|c|}{ B-splines }} & \multicolumn{7}{|c|}{ Parameter $\mu$} \\
\hline & & \multirow{2}{*}{$\frac{(\mathbf{3}, \mathbf{0})}{-2.021}$} & \multirow{2}{*}{$\frac{(3, \mathbf{1})}{-2.029}$} & \multirow{2}{*}{$\begin{array}{c}(3,2) \\
-2.023\end{array}$} & \multirow{2}{*}{$\begin{array}{r}(4,0) \\
-2.025\end{array}$} & \multirow{2}{*}{$\begin{array}{r}(\mathbf{4 , 1}) \\
-2.028\end{array}$} & \multirow{2}{*}{$\begin{array}{c}(4,2) \\
-2.032\end{array}$} & \multirow{2}{*}{$\frac{(4,3)}{-2.001}$} \\
\hline \multirow{7}{*}{ 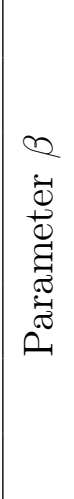 } & $(3,0$ & & & & & & & \\
\hline & $(3,1)$ & -2.228 & -2.24 & -2.266 & -2.288 & -2.298 & -2.311 & -2.298 \\
\hline & $(3,2)$ & -2.246 & -2 . & -2 & -2.2 & 7 & -2 . & -2.309 \\
\hline & $(4,0)$ & -1.967 & -1.979 & -1.979 & -1.998 & -2.002 & -2.000 & -1.987 \\
\hline & $(4,1)$ & -2.262 & -2.304 & -2.277 & -2.302 & -2.372 & -2.348 & -2.338 \\
\hline & $(4,2)$ & -2.346 & -2.243 & -2.262 & -2.300 & -2.374 & -2.357 & -2.332 \\
\hline & $(4,3)$ & -2.213 & -2.233 & -2.251 & -2.294 & -2.363 & -2.347 & -2.323 \\
\hline
\end{tabular}

Table 5: Model selection according to AIC (10 3 scale): $(r, k)$ represents the number of coefficients and the order of the B-spline.

314

2020 is obtained by choosing an approximation with 4 coefficients for the two

We fit the available data using the L-BFGS-B algorithm explained in Section 2.4.2. Figure 5 shows a very good matching between the available data and the fitting curves. To analyze the robustness of the estimate of $\alpha$ with respect to the epidemiological parameters, we perform the following analysis: choosing a grid of values based on the confidence interval of each parameter, we estimate the parameters for each possible combination and we determine the variation in the estimated value of $\alpha$, as reported in Table 6 . 


\section{It is made available under a CC-BY-NC-ND 4.0 International license.}

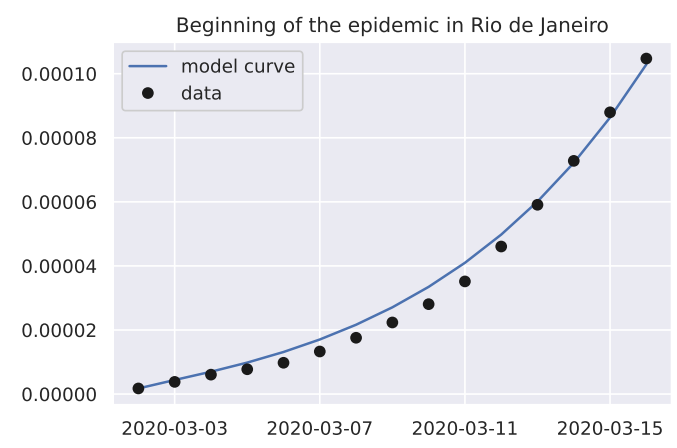

Figure 4: Fitting the curve of cumulative cases at the beginning of the outbreak
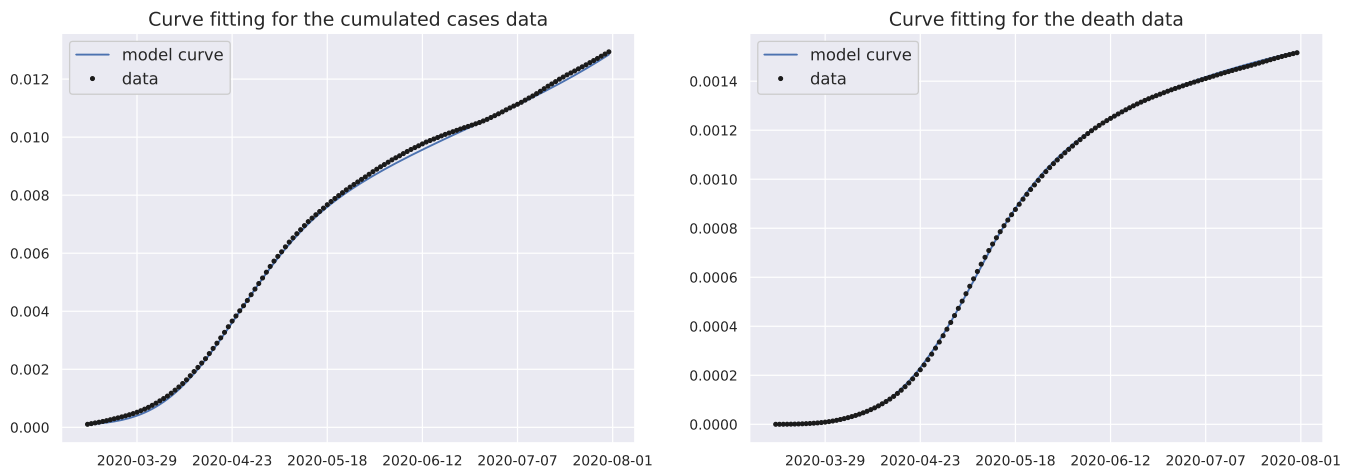

Figure 5: Fitting the curves of cumulative cases and deaths

\begin{tabular}{|c|c|c|}
\hline Parameters & Interval & Range of values of $\alpha$ \\
\hline$\tau^{-1}$ & {$[2,4]$} & {$[0.897,0.902]$} \\
\hline$\sigma^{-1}$ & {$[2,4.5]$} & {$[0.897,0.9]$} \\
\hline$\rho$ & {$\left[0,10^{-4}\right]$} & {$[0.898,0.899]$} \\
\hline$\gamma_{1}^{-1}$ & {$[6.5,9.5]$} & {$[0.894,0.903]$} \\
\hline$\gamma_{2}^{-1}$ & {$[11,16]$} & {$[0.896,0.898]$} \\
\hline
\end{tabular}

Table 6: Range of values of $\alpha$ corresponding to admissible intervals of the parameters according to the references of Table 3 

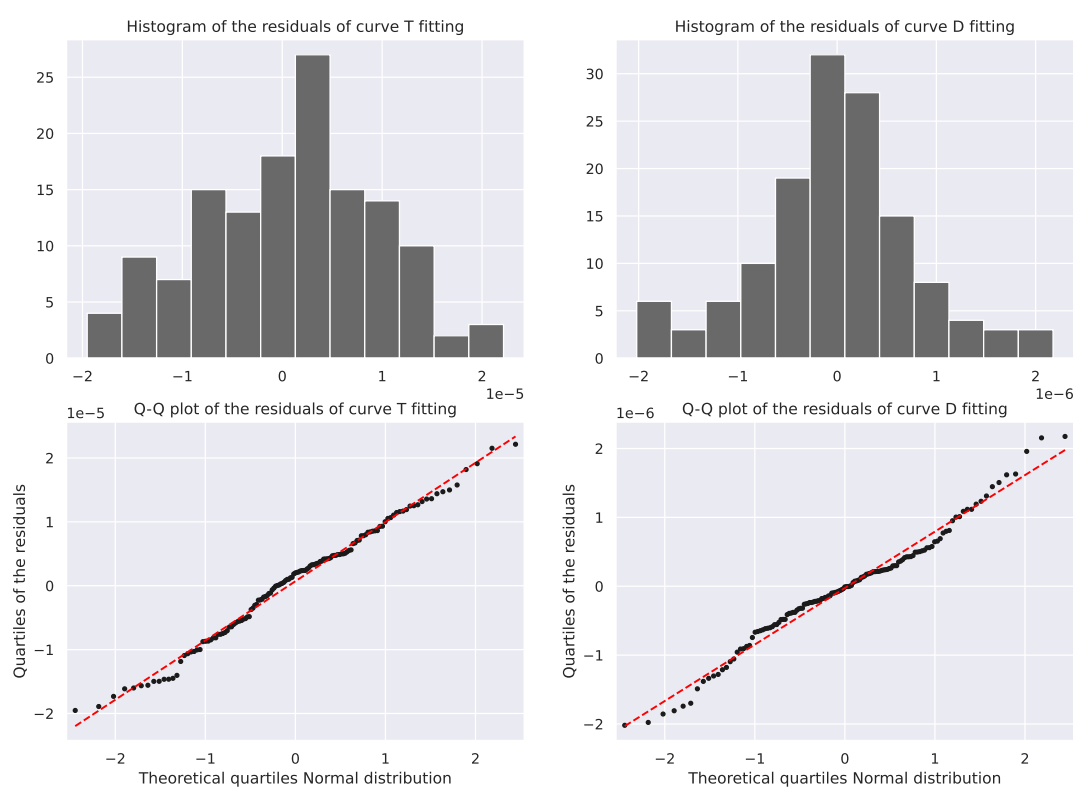

Figure 6: Graphical analysis of the model's residuals with histogram e Q-Q plot.

We also verify whether the model residuals approximate the errors, which are assumed to have a Gaussian distribution. The histograms of the residuals and the $Q-Q$ plots (comparison of the quartiles of the Gaussian distribution with the quartiles of the sampling distribution of the residuals) are shown in Figure 6. In addition to this visual analysis, we can also apply statistical tests to verify correlation and normality: the Ljung-Box test (Ljung and Box, 1978) returns a p-value 0 which indicates that the residuals are not uncorrelated; the Jarque-Bera test (Jarque and Bera, 1980) does not reject the null hypothesis at the $5 \%$ level, which is an evidence supporting the normality of the residuals.

Another important outcome of the study is the estimate of the effective (time-varying) reproductive number $\mathcal{R}_{t}$. Figure 7 describes the evolution of 
$\mathcal{R}_{t}$ in the period under consideration.

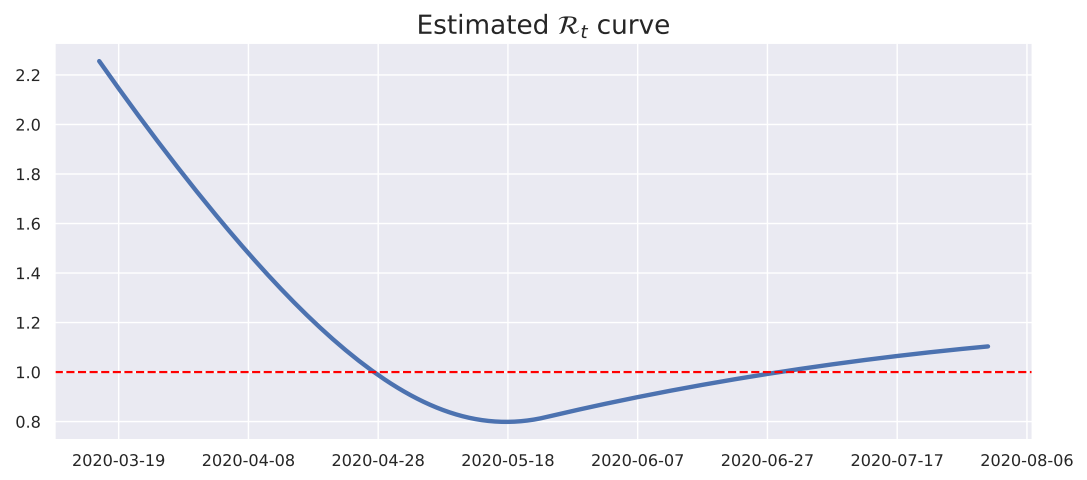

Figure 7: Effective (time-varying) reproduction number $\mathcal{R}_{t}$. The dashed red line indicates the threshold value $\mathcal{R}_{t}=1$

The correlation matrix among the estimated parameters (Section 3.3) is second coefficient of the B-spline of $\beta$ have a strong inverse correlation.

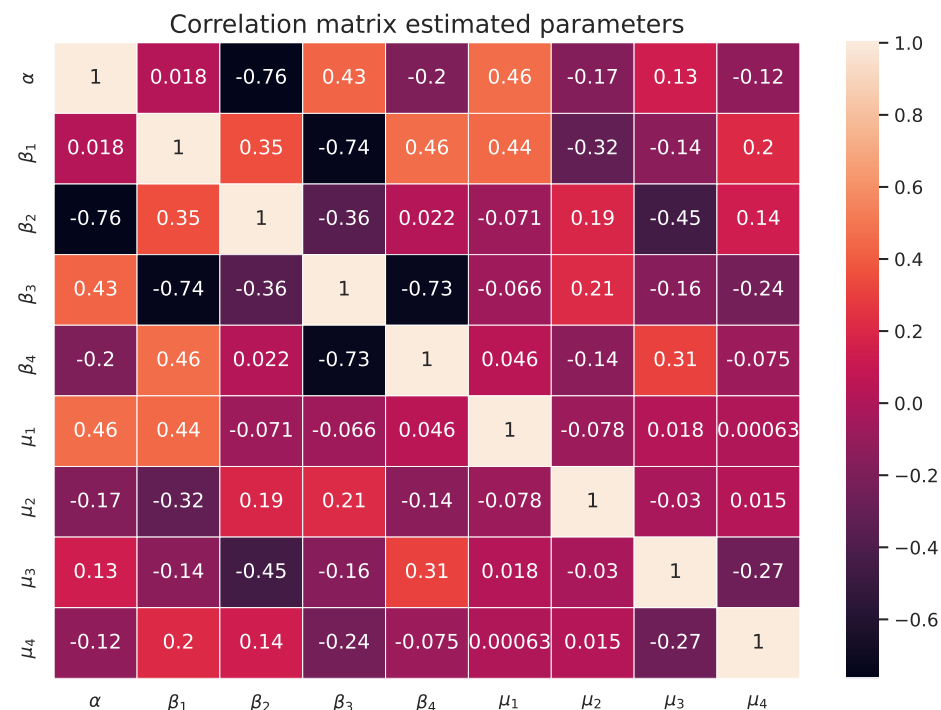

Figure 8: Correlation matrix of the estimated model's parameters. 


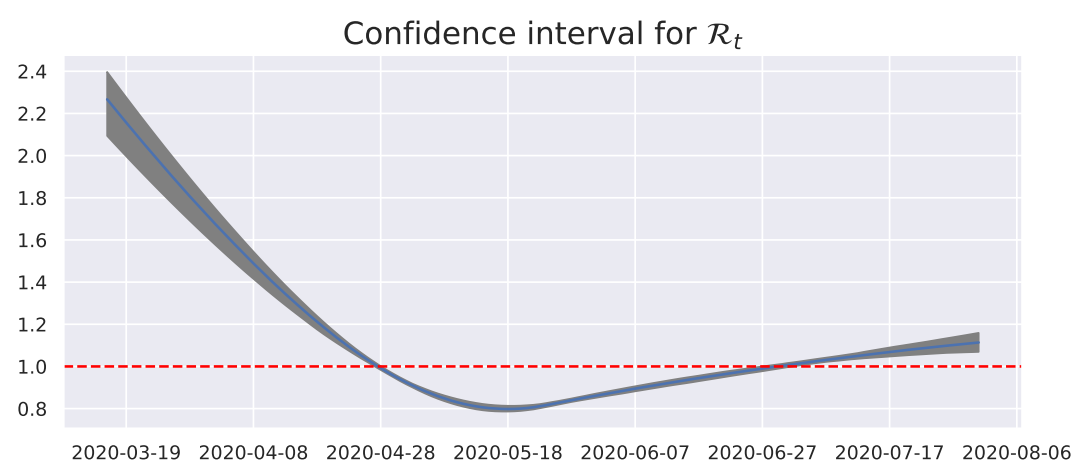

Figure 9: The blue curve is the median of the estimated curves and the gray represents the confidence interval for each time. The dashed red line is the threshold 1 for $\mathcal{R}_{t}$.

Relying on the Bootstrap method from Section 2.5 we derive confidence intervals for the parameters of the fitting: after $N=500$ simulations with $m=10$, we conclude that the $95 \%$ confidence interval for the unreported rate $\alpha$ is $(0.849,0.931)$. Figure 9 displays the confidence interval of $\mathcal{R}_{t}$ over time. Figure 10 provides a scatter plot and histogram visualization for the estimated correlations in Figure 8, Finally, Table 7 summarizes the estimates for all parameters.

\section{Discussion}

The range of values for the unreported rate $\alpha$ is in line with the results of other works on this issue: in Rio de Janeiro, (Prado et al. 2020) estimated the notification at 7.2\%; at a Brazilian national level, Canzian, 2020 Prado, 2021; Portal COVID-19 Brasil, 2021) estimated the notification of cases in the range $[7.8 \%, 8.1 \%]$.

口 It is also interesting to compare the estimates of $\mathcal{R}_{t}$ over time: in Mellan et al. 2020), the point estimate for May 9, 2020 from the state of Rio de 


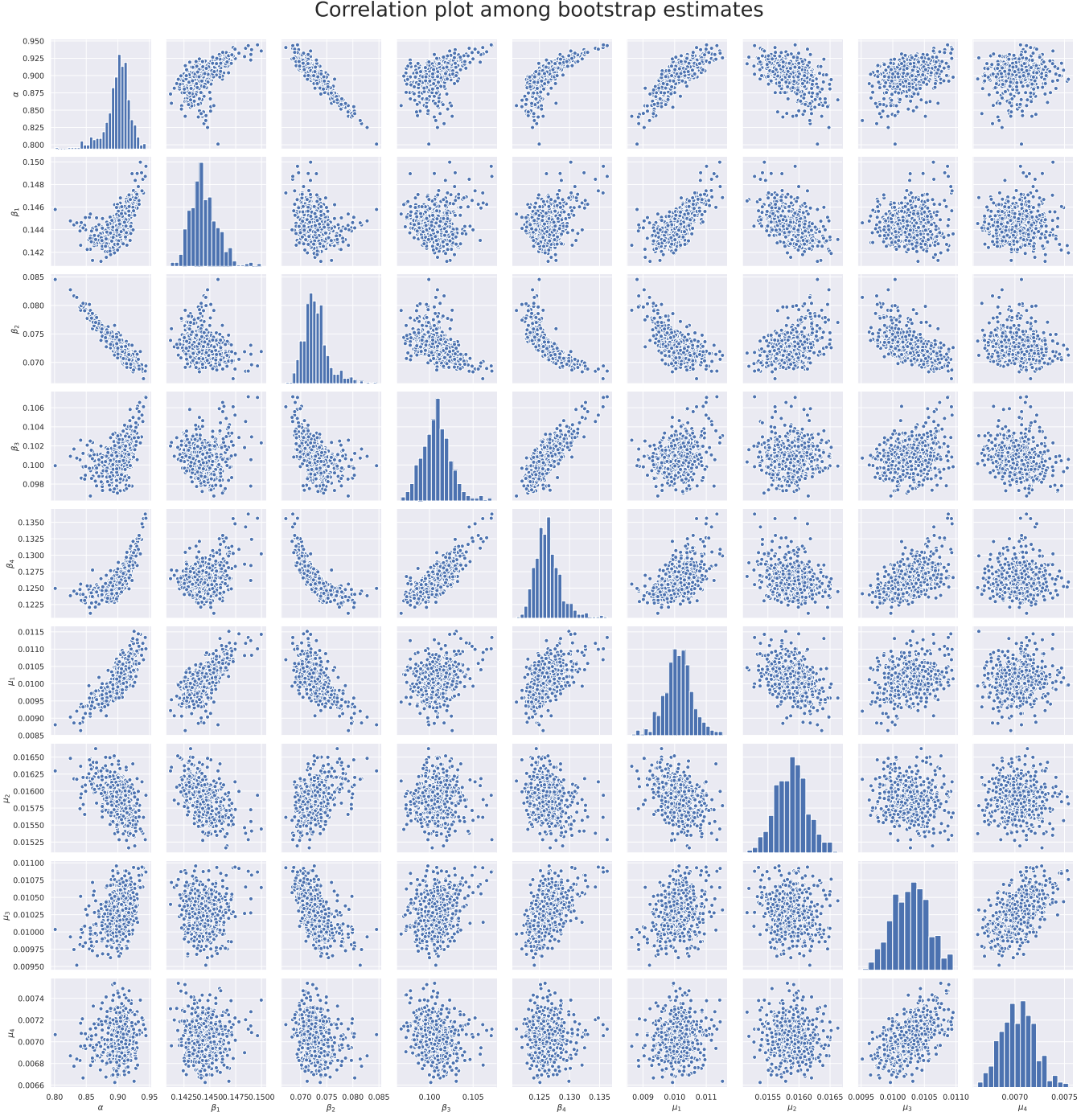

Figure 10: Scatter plots for each pair of two parameters, and their corresponding histogram. 


\begin{tabular}{|c|c|c|}
\hline Parameter & Median & Confidence interval \\
\hline$\alpha$ & 0.903 & {$[0.849,0.93]$} \\
\hline$\beta_{1}$ & 0.144 & {$[0.142,0.147]$} \\
\hline$\beta_{2}$ & 0.073 & {$[0.069,0.079]$} \\
\hline$\beta_{3}$ & 0.101 & {$[0.098,0.104]$} \\
\hline$\beta_{4}$ & 0.126 & {$[0.123,0.132]$} \\
\hline$\mu_{1}$ & 0.0101 & {$[0.0092,0.011]$} \\
\hline$\mu_{2}$ & 0.0159 & {$[0.0154,0.0164]$} \\
\hline$\mu_{3}$ & 0.0103 & {$[0.0098,0.0108]$} \\
\hline$\mu_{4}$ & $7.03 \cdot 10^{-3}$ & {$\left[6.73 \cdot 10^{-3}, 7.38 \cdot 10^{-3}\right]$} \\
\hline
\end{tabular}

Table 7: Estimates of the median and confidence intervals for each parameter.

Janeiro was 1.1 with 95\%-confidence interval $[0.9,1.3]$, which includes our range of values. The curve estimated by (Observatório COVID-19 BR, 2021) for the city of Rio de Janeiro has a very similar trend as in Figure 7 at the beginning of May, the $\mathcal{R}_{t}$ has an estimated value smaller than 1 and it grows again until it is greater than 1 at the end of July. These values corroborate our fitting.

The correlation between $\alpha$ and $\beta_{2}$ represents a limitation in terms of identifiability of the system. In order to achieve a structural identifiability result it is necessary to know the recovered curve (see Appendix B), whereas for the practical identifiability it would be useful to approximate the transmission and mortality functions with a different model. 


\section{Conclusions}

In this work, we combine tools from the analysis of differential equations, statistics, and optimization to estimate the unreported rate of COVID-19 in the city of Rio de Janeiro during the first outbreak in March-July 2020. We determine that the rate of unreported positive cases is about 90\%, with a confidence interval between $85 \%$ and $93 \%$. This means that every case reported by the health system corresponds to about 9 - 10 cases that were not detected. This estimation shall be considered as a statistical approximation of the unreported rate: in addition to the lack of testing kits during the period under evaluation, other delays and errors at all stages of the notification process may generate bad inferences. Data analysis techniques have been deployed to deal with the low quality of data.

Concerning the modeling chosen for the fitting, it is of great importance to underline that the initialization of the parameters has little influence on the final estimation of $\alpha$, supporting the robustness of our analysis. However, the fact that the residuals of the estimation are normally distributed but correlated needs more attention and will be addressed in future works.

Finally, the outcome of this work provides a description of the evolution in time of the disease reproductive number. This estimation provides a useful tool to determine periods of growth or decrease of the epidemic force at a geo-localized level, and thus to inform policymakers in their decision process to limit the spread of the virus and its consequences on the population. 


\section{Appendix A. Variance estimate}

(1) Wild, 2005, p. 21-28) given by

$$
\hat{\sigma}_{1}^{2}=\frac{1}{n-K}\left(y^{(1)}-\hat{T}(\hat{\theta})\right)^{T} \Sigma^{-1}\left(y^{(1)}-\hat{T}(\hat{\theta})\right),
$$

where $K=r+s+1$ and $n$ is the number of data points. In a similar way,

$$
\hat{\sigma}_{2}^{2}=\frac{1}{n-K}\left(y^{(2)}-\hat{D}(\hat{\theta})\right)^{T} \Sigma^{-1}\left(y^{(2)}-\hat{D}(\hat{\theta})\right) .
$$

\section{Appendix B. DAISY}

DAISY - Differential Algebra for Identifiability of SYstems - is a software based on the programming language Reduce, specific to the problem of identifiability in dynamical systems. DAISY requires certain specific conditions on the system, that allow to apply algebraic methods to the model under consideration. In order to rewrite our system (1) in terms of the DAISY algorithm, we treat all model parameters' in Section 2.1 as constant in time. If we assume to know the curve $R$ of recovered cases, we verify that model (1) is globally identifiable. However, the knowledge of $R$ may sometimes be only partially available, as discussed in Section 2.2. Without access to these data, DAISY has not been able to certify the structural identifiability of the system.

\section{Acknowledgements}

The authors wish to thank Luiz Max Carvalho (FGV EMAp) and Marcelo Fernandes (FGV EESP) for fruitful discussions about several statistical meth- 
ods applied in this research.

The first and third authors were supported by FAPERJ and CNPq, Brazil.

The second author acknowledges the funding of the Natural Sciences and Engineering Research Council of Canada (NSERC). The third author thanks the Center for the Development of Mathematics and Science (FGV CDMC) for their support.

\section{References}

Aronna, M., Guglielmi, R., Moschen, L., 2021. A model for COVID-19 with isolation, quarantine and testing as control measures. Epidemics 34, 100437. URL: https : / / wWw . sciencedirect . com/science/article/ pii/S1755436521000025, doi:https : / / doi .org/10 .1016/j . epidem . 2021.100437.

Audoly, S., Bellu, G., D’Angio, L., Saccomani, M., Cobelli, C., 2001. Global identifiability of nonlinear models of biological systems. IEEE Transactions on Biomedical Engineering 48, 55-65. doi:10.1109/10.900248.

Bellu, G., Saccomani, M.P., Audoly, S., D’Angiò, L., 2007. DAISY: A new software tool to test global identifiability of biological and physiological systems. Computer Methods and Programs in Biomedicine 88, 52-61.

Byrd, R.H., Lu, P., Nocedal, J., Zhu, C., 1995. A limited memory algorithm for bound constrained optimization. SIAM Journal on Scientific Computing 16, 1190-1208.

Byrne, A.W., McEvoy, D., Collins, A.B., Hunt, K., Casey, M., Barber, A., Butler, F., Griffin, J., Lane, E.A., McAloon, C., et al., 2020. Inferred 
duration of infectious period of SARS-CoV-2: rapid scoping review and analysis of available evidence for asymptomatic and symptomatic COVID19 cases. BMJ Open 10, e039856.

Canzian, F., 2020. Estados e municípios no país relatam subnotificação gigantesca de casos (States and cities alert on a huge underreporting of cases). Available at https : / / www1 . folha . uol . com . br / equilibrioesaude / 2020/04/estados-e-municipios-no-pais-relatam-subnotificacaogigantesca-de-casos.shtml. Folha de São Paulo Newspaper.

Cao, J., Huang, J.Z., Wu, H., 2012. Penalized nonlinear least squares estimation of time-varying parameters in ordinary differential equations. Journal of Computational and Graphical Statistics 21, 42-56.

Croda, J., Oliveira, W.K.d., Frutuoso, R.L., Mandetta, L.H., Baia-da Silva, D.C., Brito-Sousa, J.D., Monteiro, W.M., Lacerda, M.V.G., 2020. COVID19 in Brazil: advantages of a socialized unified health system and preparation to contain cases. Revista da Sociedade Brasileira de Medicina Tropical 53.

Dantas, G., Siciliano, B., França, B.B., da Silva, C.M., Arbilla, G., 2020. The impact of COVID-19 partial lockdown on the air quality of the city of Rio de Janeiro, Brazil. Science of the Total Environment 729, 139085.

De Boor, C., 1978. A practical guide to splines. volume 27. Springer Verlag New York.

Efron, B., Tibshirani, R., 1986. Bootstrap methods for standard errors, 
confidence intervals, and other measures of statistical accuracy. Statistical Science , 54-75.

Estadão, 2020. Governo do Rio cria classificação em 3 bandeiras para flexibilizar isolamento (Government of Rio creates classification in 3 flags to make isolation more flexible). Available at https://revistapegn.globo. com / Noticias / noticia / 2020 / 05 / pegn - governo - do - rio - cria classificacao-em-3-bandeiras-para-flexibilizar-isolamento . html.

IBGE, 2020. Brazilian Institute for Geography and Statistics. Brazilian National Household Survey Sample - PNAD COVID-19. Available at https://www.ibge.gov.br/estatisticas/sociais/trabalho/27946divulgacao-semanal-pnadcovid1.html?=\&t=downloads.

IBGE, 2021. Brazilian Institute for Geography and Statistics. Cities and States. Available at https://www.ibge.gov.br/cidades-e-estados/ rj/rio-de-janeiro.html.

Jarque, C.M., Bera, A.K., 1980. Efficient tests for normality, homoscedasticity and serial independence of regression residuals. Economics Letters 6, $255-259$.

Joshi, M., Seidel-Morgenstern, A., Kremling, A., 2006. Exploiting the bootstrap method for quantifying parameter confidence intervals in dynamical systems. Metabolic Engineering 8, 447-455.

Kucirka, L.M., Lauer, S.A., Laeyendecker, O., Boon, D., Lessler, J., 2020. Variation in false-negative rate of reverse transcriptase polymerase chain 
reaction-based SARS-CoV-2 tests by time since exposure. Annals of Internal Medicine 173, 262-267.

Li, R., Pei, S., Chen, B., Song, Y., Zhang, T., Yang, W., Shaman, J., 2020. Substantial undocumented infection facilitates the rapid dissemination of novel coronavirus (SARS-CoV-2). Science 368, 489-493.

Liang, H., Miao, H., Wu, H., 2010. Estimation of constant and time-varying dynamic parameters of HIV infection in a nonlinear differential equation model. The Annals of Applied Statistics 4, 460.

Ljung, G.M., Box, G.E., 1978. On a measure of lack of fit in time series models. Biometrika 65, 297-303.

Ljung, L., Glad, T., 1994. On global identifiability for arbitrary model parametrizations. Automatica 30, 265-276.

Mellan, T.A., Hoeltgebaum, H.H., Mishra, S., Whittaker, C., Schnekenberg, R.P., Gandy, A., Unwin, H.J.T., Vollmer, M.A., Coupland, H., Hawryluk, I., et al., 2020. Subnational analysis of the COVID-19 epidemic in Brazil. MedRxiv .

Miao, H., Xia, X., Perelson, A.S., Wu, H., 2011. On identifiability of nonlinear ODE models and applications in viral dynamics. SIAM Review 53, 3-39.

Moschen, L.M., 2021. Repository COVID-19. Github. Available at https : //github.com/lucasmoschen/covid-19-model. 
Municipal Health Department, City Hall of Rio de Janeiro, 2021. Dados individuais dos casos confirmados de COVID-19 no município do Rio de Janeiro (Individual data of COVID-19 confirmed cases in the city of Rio de Janeiro). Available at https : //www . arcgis . com/home/item . html? $i d=f 314453 b 3 a 55434 e a 8 c 8 e 8 c a a a 2 d 8 d b 5$.

Nogrady, B., 2020. What the data say about asymptomatic COVID infections. Nature .

Observatório COVID-19 BR, 2021. R efetivo no Rio de Janeiro (Effective $\mathrm{R}$ in Rio de Janeiro. Available at https : / / covid19br . github . io / municipios .html?aba=aba3\&uf=RJ\&mun=Rio_de_Janeiro.

Official Journal of the State of Rio de Janeiro, 2020a. Ordinance number 46.973, March 16th 2020. Available at https://pge.rj.gov.br/comum/ code/MostrarArquivo .php?C=MTAyMjI.

Official Journal of the State of Rio de Janeiro, 2020b. Law number 8859, June 3rd, 2020. Available at http://www . aeerj . net . br/file/04-062020-leiestadomascara.pdf.

Portal COVID-19 Brasil, 2021. COVID-19 BRASIL. Available at https : //ciis.fmrp.usp.br/covid19/.

Prado, M.F.d., Antunes, B.B.d.P., Bastos, L.d.S.L., Peres, I.T., Silva, A.d.A.B.d., Dantas, L.F., Baião, F.A., Maçaira, P., Hamacher, S., Bozza, F.A., 2020. Análise da subnotificação de COVID-19 no Brasil (Analysis of COVID-19 underreporting in Brazil). Revista Brasileira de Terapia Intensiva . 
Prado, M.F.d.et al.., 2021. Análise de subnotificação do número de casos confirmados da COVID-19 no Brasil (Analysis of underreporting of the number of confirmed cases of COVID-19 in Brazil). Available at https : //drive.google.com/file/d/1_whlqZnGgvqHuWCG4-JyiL2X9WXpZAe3/ view.

Rai, B., Shukla, A., Dwivedi, L.K., 2021. Incubation period for COVID-19: a systematic review and meta-analysis. Journal of Public Health , 1-8.

Ramsay, J.O., Hooker, G., Campbell, D., Cao, J., 2007. Parameter estimation for differential equations: a generalized smoothing approach. Journal of the Royal Statistical Society: Series B (Statistical Methodology) 69, 741-796.

Saccomani, M.P., Thomaseth, K., 2019. Calculating all multiple parameter solutions of ODE models to avoid biological misinterpretations. Mathematical Biosciences and Engineering 16, 6438-6453.

Seber, G., Wild, C., 2005. Nonlinear Regression. Wiley Series in Probability and Statistics, Wiley. URL: https://books.google.com.br/books?id= YBYICpBNo_cC.

The 2019 nCoV Outbreak Joint Field Epidemiology Investigation Team and Q. Li, 2020. An Out-break of NCIP (2019-nCoV) Infection in China Wuhan, Hubei Province, 2019 - 2020. Available at http : / / weekly . chinacdc . cn / en / article / id / e3c63ca9 - dedb - 4fb6 - 9c1c d057adb77b57.

Virtanen, P., Gommers, R., Oliphant, T.E., Haberland, M., Reddy, T., Cournapeau, D., Burovski, E., Peterson, P., Weckesser, W., Bright, J., 
540 et al., 2020. SciPy 1.0: fundamental algorithms for scientific computing in

${ }_{541}$ Python. Nature Methods 17, 261-272.

${ }_{542}$ World Health Organization, 2020. Coronavirus disease (COVID-19). Avail543 \able at https : / / www . who . int / emergencies / diseases / novel 544 coronavirus - 2019 / question - and - answers - hub / q - a - detail /

545 Coronavirus-disease-covid-19. 